\title{
Vector Flow Mapping Using Plane Wave Ultrasound Imaging
}

\author{
Sarah Dort ${ }^{1}$, Stephan Muth ${ }^{1}$, Abigail Swillens ${ }^{2}$, Patrick Segers ${ }^{2}$, Guy Cloutier ${ }^{3}$ and Damien Garcia ${ }^{1}$ \\ ${ }^{1}$ Research Unit of Biomechanics and Imaging in Cardiology, University of Montreal Hospital Research Center, Canada \\ ${ }^{2}$ Institute of Biomedical Technology, University of Ghent, Belgium \\ ${ }^{3}$ Laboratory of Biorheology and Medical Ultrasonics, University of Montreal Hospital Research Center, Canada \\ Email: damien.garcia@crchum.qc.ca; Website: www.biomecardio.com
}

\begin{abstract}
Conventional pulse wave Doppler techniques can only provide one-dimensional blood velocity components parallel to the direction of the beam and conventional focusing provides limited frame rates of about 30-40 frames per second. As a solution to these well known limitations we perform a two-dimensional vector mapping using compounded coplanar oriented plane waves, analogous to vector-Doppler. Our method was tested using Field II simulations of both stationary parabolic pipe flow and computational fluid dynamics determined flow through a patient specific carotid artery. Our results show the ability for this method to provide more discernible representation of the flow dynamics compared with conventional color-Doppler imaging, while maintaining a frame rate of roughly 500 frames per second. Quantitative comparison with known velocity fields provides robust validation and demonstrates error comparable to that found in literature using conventional Doppler measurements. Moreover, this method provides a promising means to quantify quick transitory events and complex flow structures unattainable with clinical color-Doppler.
\end{abstract}

Keywords: blood flow, ultrafast ultrasound, plane wave imaging, vector flow

\section{INTRODUCTION}

Color-Doppler imaging (CDI) is the most widely used clinical tool to non-invasively evaluate vascular and cardiac function through blood velocity estimation. Clinical CDI systems work by emitting multiple periodic focused ultrasound pulses into a tissue medium to cover a region of interrogation. Standard post-processing dictates the calculation of velocity components in-line with the beam direction [1].

Despite the efficacy of CDI, this technique retains some well known drawbacks; conventional CDI has a limited frame rate and the error in the blood velocity estimation is dependent on the angle of acquisition. To elaborate, in order to image a full area of interest, multiple focused scans are acquired to form one frame. Therefore, the frame rate is not only dependent on the depth of scan and speed of acoustic propagation but also on the number of scan-lines required for an adequate field of view. This creates compromising between the spatial resolution and the temporal resolution. Additionally, orthogonal components of velocity to the beam direction cannot be detected using conventional CDI. Thus, a
color-Doppler image does not contain a sufficient amount of data to allow full and accurate quantification of the hemodynamics [2].

Recently, some groups have showed interest in plane wave imaging as a solution to the limited frame rate of customary ultrasound [3,4]. In plane wave imaging, one single pulse emission event is used to illuminate the medium rather than multiple focused beams. This technique effectively decreases the frame rate by a factor of the number of scan-lines acquired for a focused image. Although the resulting plane wave images have lower quality contrast and spatial resolution than their conventional counterpart, one study [3] showed that focused quality can be achieved by coherently compounding multiple plane wave images, while still maintaining a frame rate of at least 10 times more rapid.

To address the angular dependence of CDI, many methods of multi-dimensional estimation of flow velocity have been proposed to provide better quantification for complex flow patterns [2, 5, 6]. One major method is Vector Doppler. Vector Doppler, an elaboration on conventional onedimensional (1D) scans, consists in capturing two or more series of coplanar Doppler images and recomposing their velocity components in a common coordinate system. In this way, a vector map of the hemodynamics can be achieved in two dimensions (2D).

In this work, we propose a method of vector flow mapping using plane wave ultrasound imaging to address the aforementioned limitations. Using angled coplanar plane wave images in an alternating manner we apply a series of post-processing steps to obtain a $2 \mathrm{D}$ vector map, analogous to vector Doppler; however, we achieve a significantly elevated frame rate. This methodology was robustly validated on both ultrasound simulations of simple stationary pipe flow and more complex flow patterns in the carotid arteries. Our approach demonstrates more coherent depiction of flow than clinical color-Doppler with high frame rates and thus the opportunity to quantify fast transitory events occurring during the cardiac cycle which would not otherwise be captured. As atherosclerotic development has been associated to areas such as the carotid arteries which possess intricate flow patterns with flow separations, a technique such as this would be of high interest for clinical application. 


\section{METHODOLOGY}

\section{A. Ultrasound Simulations}

Using the Field II software, images of blood vessels were simulated with a stationary pipe flow phantom and a pulsatile carotid artery phantom. Acquisitions were taken using a simulated 128 element $\mathrm{L} 7-4$ transducer with a $5 \mathrm{MHz}$ central frequency and acoustic propagation of $1540 \mathrm{~m} / \mathrm{s}$. Consecutively, $N$ tilted plane waves were emitted to allow for compounding to form each oriented frame at angle $\beta$. To this end, a linear delay was applied upon emission to form the titled planes at angles $\alpha$, with no focusing used in emission or reception, as demonstrated in fig. 1. Tissue medium was modeled by point scatterers with a random distribution using 10 scatterers per resolution cell possessing normally distributed amplitudes.

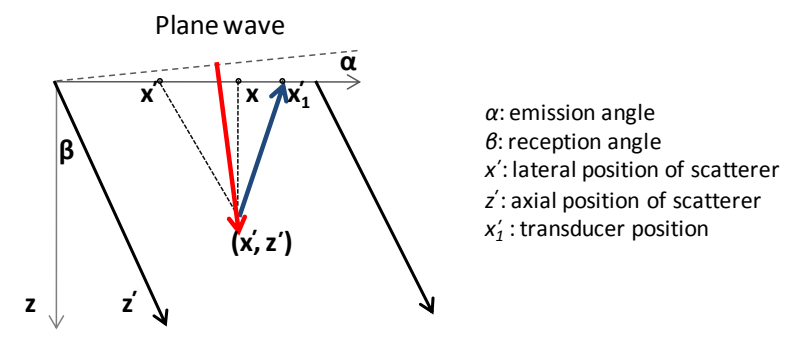

Figure 1. Representation of emission (red arrow) and reception (blue arrow) at angles $\alpha$ and $\beta$.

First, stationary pipe flow was modeled through a straight tube at a depth of $20 \mathrm{~cm}$. For each successive frame, the propagation of the point scatterers followed the Poiseuille equation:

$$
v(R)=v_{\max }\left(1-\left(R / R_{\max }\right)^{n}\right),
$$

where $v$ indicates the velocity as a function of radial position $R$, Rmax denotes the radius of the vessel (4 mm), Vmax is the maximum velocity $(80 \mathrm{~cm} / \mathrm{s})$ with $\mathrm{n}=2$ the shape factor indicating a parabolic flow profile.

Subsequently, a more elaborate computational fluid dynamics (CFD) simulation environment was used to model blood flow through a carotid bifurcation developed in [7]. The numerical simulation was performed using the commercial software Fluent 6.2 (Ansys Inc., Canonsburg, PA) for a patient specific three-dimensional (3D) model extracted from 40 MR scans (Mimics, Leuven, Belgium) and a pulse wave Doppler measured velocity profile. The measured profile was applied at the common carotid artery and a partition of 40 and 60 percent of the flow was applied to the division entering the external and internal carotid branches. Using the positional blood velocity data obtain from the CFD simulations, the location of each point scatterers was updated in each successive frame.

\section{B. Post processing of plane wave images}

In order to process the echo signals in each plane wave image, for each point $\left(\mathrm{x}_{\mathrm{p}}^{\prime}, \mathrm{z}_{\mathrm{p}}^{\prime}\right)$ we summed the contribution of each tranducer element determined by a certain travel time $\tau$ which represents the time taken to reach the point and return to the transducer, shown in fig. 1 and demonstrated in (2) and (3). Using geometric relations, the time taken for a wave to encounter each point is given in the following equation:

$$
\tau_{e m}=\left(\cos (\alpha-\beta) \cdot z^{\prime}+\sin \alpha \cdot x^{\prime}\right) / c,
$$

where, $\alpha$ and $\beta$ are the emission and reception angles, respectively, and $x^{\prime}$ and $z^{\prime}$ represent any given point in the angled coordinate system. The travel times in reception can be calculated with the following equation:

$$
\tau_{r e c}=\sqrt{z^{\prime 2}+\left(x_{1}^{\prime}-x^{\prime}\right)^{2}+2 \sin \beta \cdot z^{\prime} \cdot\left(x^{\prime}-x_{1}^{\prime}\right)} / c,
$$

where, $x_{1}{ }^{\prime}$ represents the transducer location. By adding equations 2 and 3 , the total travel time $\tau$ is:

$$
\tau=\tau_{\text {em }}+\tau_{\text {rec }} .
$$

The delay and sum technique using (4) permits the procurement of the correct position and amplitude of the scatterers within the medium in an angled coordinate system displayed in fig. 2. Additionally, $N$ tilted plane waves were compounded per oriented frame.

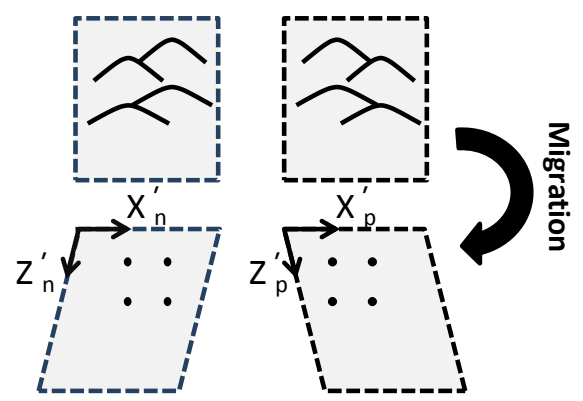

Figure 2. Delay and sum method in an oriented coordinate system.

\section{2D Velocity Vector Reconstruction}

Analogous to color-Doppler, 1D components of the velocity were calculated with respect to the oriented direction $z$ ' using a frequency domain based cross correlation.

The $2 \mathrm{D}$ vector field reconstruction was done using the two sets of oriented velocity information, shown in fig. 3, and recomposing them into a common Cartesian coordinate system using the following two equations:

$$
V_{x}=\frac{V_{1}-V_{2}}{2 \sin \beta} \quad \text { and } \quad V_{z}=\frac{V_{1}+V_{2}}{2 \cos \beta},
$$

where $V_{\mathrm{x}}$ and $V_{\mathrm{z}}$ are the lateral and axial components of the $2 \mathrm{D}$ velocity, respectively. $V_{1}$ and $V_{2}$ represent the $1 \mathrm{D}$ velocity components of the flow parallel to the ultrasound beam determined from acquisitions 1 and 2 , at angle $\pm \beta$. Finally, a discrete cosine transform based penalized least squares method of regularization was applied to the reconstruction velocity field to smooth aberrant vectors [8]. 


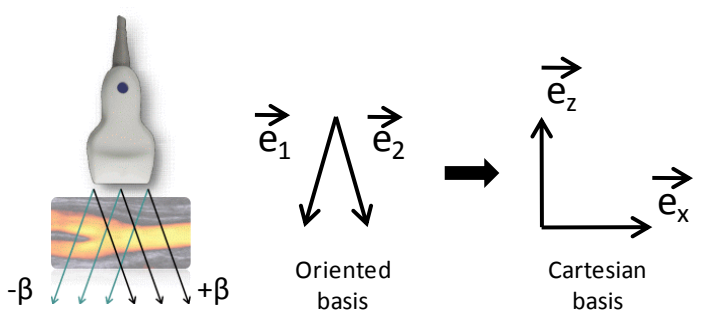

Figure 3. Coplanar oriented acquisitions resolved in common basis.

\section{RESULTS}

\section{A. Pipe flow phantom}

For the pipe flow phantom, a pulse repetition frequency (PRF) of $10 \mathrm{kHz}$ was used to acquire five tilted plane wave sequences used to compound each oriented image at $\beta$ equal to $\pm 15^{\circ}$, allowing for an overall $2 \mathrm{D}$ vector map frame rate of roughly $500 \mathrm{fps}$. The results of our reconstruction method on the Field II simulations for stationary parabolic pipe flow are demonstrated in figs. 4 and 5. From fig. 4 , we see the qualitative ability for the methodology to provide a coherent estimation of the theoretical flow. The estimation depicts a parabolic profile with relevant magnitude.

Figure 5, gives a more quantitative evaluation of the performance of this methodology. In the lateral component of velocity $V_{x}$, there is a clear underestimation, which is concurrent to the results found in [9]. As for the axial velocity component $V_{z}$, there is a better proclivity to follow more
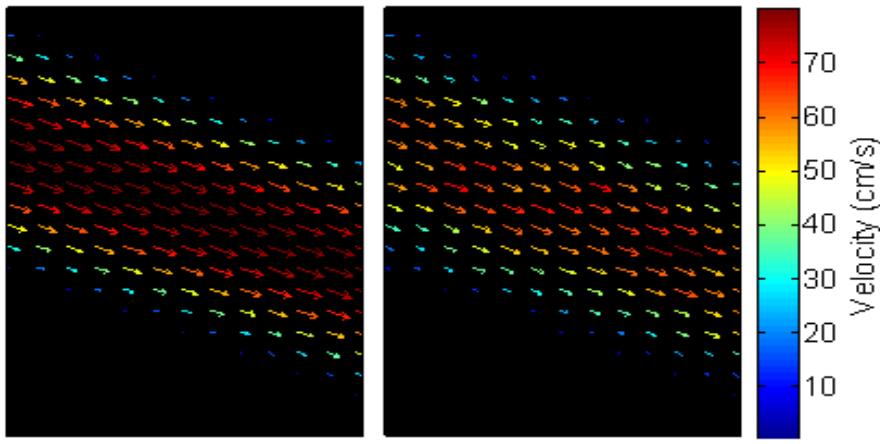

Figure 4. Magnitude and direction of (left) true velocity field defined by

(1) and (right) estimated velocity field using plane wave 2D reconstruction
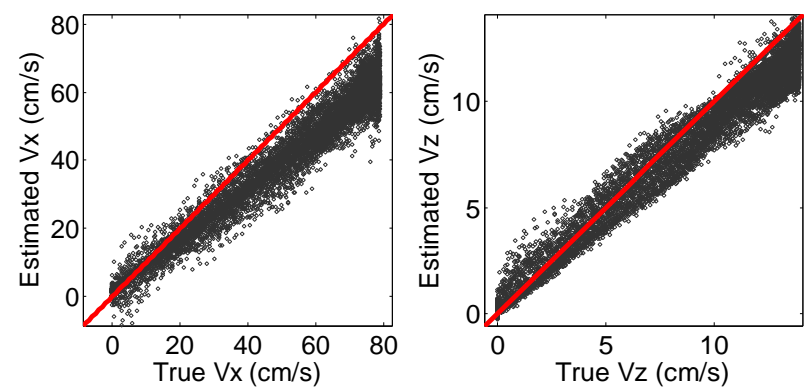

Figure 5 . True velocity compared with estimated velocity at all points in the pipe flow for (left) lateral and (right) axial components. closely to the red line indicating zero error.

Additionally, we calculated the normalized root mean squared error (NRMSE) between the true and estimated velocities for each point over the image using the following equation:

$$
N R M S E=\sqrt{\sum\left|V_{\text {true }}-V_{\text {est }}\right|^{2}} / \sqrt{\sum\left|V_{\text {true }}\right|^{2}} .
$$

The lateral and axial components were found to have 22.3 and 13.1 percent NRMSE, which is also in agreement with the results found in [9].
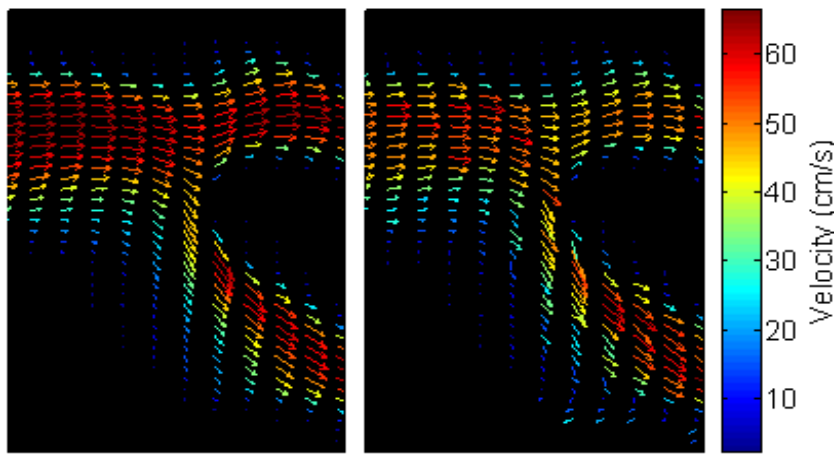

Figure 6. Magnitude and direction of (left) true velocity field in carotid bifurcation and (right) estimated velocity field using plane wave $2 \mathrm{D}$ reconstruction.

\section{B. Carotid flow phantom}

Similarly, concerning the flow through the simulated carotid artery, a PRF of $10 \mathrm{kHz}$ was used to acquire five tilted plane wave sequences for the compounding of each image oriented at $\beta$ equal to $\pm 15^{\circ}$ with a resulting frame rate of 500 fps. Shown in fig. 6 is the vector mapping of the carotid bifurcation. Again, on this more complex velocity field, our methodology is able to reconstruct the flow pattern with good agreement to the CFD reference flow. Akin to the results of the pipe flow phantom, we can observe an underestimation in the lateral component of the velocity, particularly in the external branch of the carotid, where the flow runs orthogonal to the imaging direction.
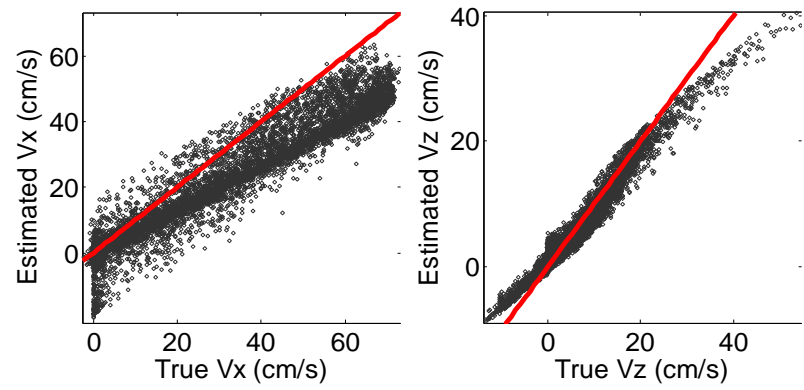

Figure 7 . True velocity compared with estimated velocity at all points in the carotid flow for (left) lateral and (right) axial components. 
In comparing the estimated lateral component of velocity to the reference velocity, there is a similar trend of slight underestimation with a coinciding amount of variation, shown in fig. 7. The axial component estimation shows better agreement with the reference CFD velocities. The NRMSE was found to be 29.8 and 21.2 percent for the lateral and axial components, respectively.

\section{DISCUSSION AND CONCLUSION}

In this study, we proposed a $2 \mathrm{D}$ vector flow mapping from plane wave ultrasound imaging, in order to create an alternative method to improve blood flow imaging. As demonstrated in our results, we were able to represent complex flow patterns in a more comprehensible manner than that of standard CDI. Additionally, with acceptable errors similar to results found in [9] using conventional vector Doppler, we were able to reconstruct velocity fields with a frame rate of roughly $500 \mathrm{fps}$ compared to 30 to $40 \mathrm{fps}$ for that of CDI. This significant increase in frame rate creates the possibility of providing better quantification of fast transitory events occurring during the cardiac cycle which could not otherwise be detected with standard scans.

Furthermore, contrary to CDI whose quantification is exhibited in a color coded image with intensity scales describing the velocity with respect to the beam direction, 2D vector mapping provides axial and lateral components of the flow. This enables a more intuitive representation of the flow and permits us to compute other important characteristics of the flow such as vorticity and circulation.

Finally, due to the capability of the CFD coupled ultrasound simulation environment to allow comparisons between known complex flow patterns and the reconstructed estimation using our methodology, we are able to provide robust quantitative evaluation of this method for clinical application. Nonetheless, our results are based on simulations thus extension will be made to in vitro and in vivo applications of this method.

\section{ACKNOWLEGDEMENTS}

This work was supported by MEDITIS funded by NSERC (Natural Science and Engineering Council of Canada). We thank Abigail Swillens and Patrick Segers for contributing their computational fluid dynamics coupled ultrasound simulations and Lasse Løvstakken for contributing the MR scans.

\section{REFERENCES}

[1] D. H. Evans, W. N. McDickens, R. Skidmore, and J. P. Woodcock, Doppler ultrasound: physics, instrumentation, and clinical applications: John Wiley \& Sons, 1991.

[2] M. Arigovindan, M. Suhling, C. Jansen, P. Hunziker, and M. Unser, "Full motion and flow field recovery from echo Doppler data," IEEE Transactions on Medical Imaging, vol. 26, pp. 31-45, 2007.

[3] G. Montaldo, M. Tanter, J. Bercoff, N. Benech, and M. Fink, "Coherent plane-wave compounding for very high frame rate ultrasonography and transient elastography," IEEE Transactions on Ultrasonics Ferroelectrics, and Frequency Control, vol. 56, pp. 489-506, 2009.

[4] J. Bercoff, G. Montaldo, T. Loupas, D. Savery, F. Meziere, and M. Fink, "Ultrafast compounding Doppler imaging: providing full blood flow characterization " IEEE Transactions on Ultrasonics, Ferroelectrics, and Frequency Control, vol. 58, pp. 134-147, 2011.

[5] B. Dunmire, K. W. Beach, L.-H. Labs, M. Plett, and D. E. Strandness, "Cross-beam vector Doppler ultrasound for angle-independent velocity measurements," Ultrasound in Medicine \& Biology, vol. 26, pp. 1213-1235, 2000.

[6] L. Capineri, M. Scabia, and L. Masotti, "A Doppler system for dynamic vector velocity maps," Ultrasound in Medicine \& Biology, vol. 28, pp. 237248, 2002.

[7] A. Swillens, L. Lovstakken, J. Kips, H. Torp, and P. Segers, "Ultrasound simulation of complex flow velocity fields based on computational fluid dynamics," IEEE Transactions on Ultrasonics, Ferroelectrics and Frequency Control, , vol. 56, pp. 546-556, 2009.

[8] D. Garcia, "Robust smoothing of gridded data in one and higher dimensions with missing values," Computational Statistics \& Data Analysis, vol. 54, pp. 1167-1178, 2010.

[9] A. Swillens, P. Segers, H. Torp, and L. Lovstakken, "Two-dimensional blood velocity estimation with ultrasound: speckle tracking versus crossed-beam vector Doppler based on flow simulations in a carotid bifurcation model," IEEE Transactions on Ultrasonics, Ferroelectrics, and Frequency Control, vol. 57, pp. 327-339, 2010. 